\title{
Fitting along the Fundamental Plane: New comparisons of jet physics across the black hole mass scale
}

\author{
Sera Markoff ${ }^{1}$, Michael A. Nowak ${ }^{2}$, Dipankar Maitra ${ }^{3}$, Jörn Wilms ${ }^{4}$, \\ Elena Gallo ${ }^{3}$, Robert Hynes ${ }^{5}$ and Richard Plotkin ${ }^{1}$ \\ ${ }^{1}$ Astronomical Institute "Anton Pannekoek", University of Amsterdam \\ Science Park 904, 1098XH, Amsterdam, the Netherlands \\ email: s.b.markoff,r.m.plotkin@uva.nl \\ ${ }^{2}$ Kavli Institute for Astrophysics and Space Research \\ Cambridge, MA 02139, USA \\ email: mnowak@space.mit.edu \\ ${ }^{3}$ Department of Astronomy, University of Michigan \\ Ann Arbor, MI 48109, USA \\ email: dmaitra, egallo@umich.edu \\ ${ }^{4}$ Dr. Karl Remeis-Observatory \& ECAP, University of Erlangen-Nuremberg, Bamberg, \\ Germany \\ email: joern.wilms@sternwarte.uni-erlangen.de \\ ${ }^{5}$ Department of Physics and Astronomy, Louisiana State University, Baton Rouge, LA 70803 \\ email: rih@theory.phys.lsu.edu
}

\begin{abstract}
Correlations between the radio and X-ray bands in the hard state of black hole X-ray binaries (BHBs) have led to the discovery of the Fundamental Plane of black hole accretion, linking accretion-driven radiative attributes to black hole mass. Although this discovery has led to new constraints on radiative efficiencies, there is still significant degeneracy in terms of understanding the governing physics. I present several new results exploring the processes driving the Fundamental Plane over the black hole mass range. These include the first ever homogeneous fits of sources at approximately the same Eddington luminosity but millions of times different in mass, which I focus on for this proceeding article.
\end{abstract}

Keywords. black hole physics; X-rays: binaries; galaxies: jets; accretion, accretion disks; radiation mechanisms: nonthermal

\section{Introduction}

By now the existence of a Fundamental Plane of black hole $(\mathrm{BH})$ accretion has been established with high statistical significance (e.g., Merloni et al. 2003; Falcke et al. 2004; Körding et al. 2006). In observational terms, the Fundamental Plane (FP) can be described as a log-linear correlation between the radio and X-ray luminosities of both stellar mass and supermassive BHs, with a mass dependent normalization term between them. This behavior, which holds over orders of magnitude in power, indicates that accreting BHs experience at least one mode in which they regulate their radiative and mechanical luminosity similarly at a given Eddington accretion rate $\dot{m}=\dot{M} / \dot{M}_{\mathrm{Edd}}$, regardless of their mass.

The regression methods used to derive the coefficients of the FP generally result in significant scatter around the correlation, anywhere from $\sim 0.38-0.65$ dex, depending on the sample of active galactic nuclei (AGN) used (Körding et al. 2006). While new results on the intrinsic behavior of $\mathrm{BH}$ binaries (BHBs) (where the radio/X-ray correlation was 
originally discovered, see, e.g., Corbel et al. 2003) have revealed deviations from a perfect power-law (see Coriat et al., this volume), the scatter could also be due to the sample selection. One major difference between the samples used in the two FP "discovery" papers is that Merloni et al. (2003) included Seyferts while Falcke et al. (2004) did not. If one believes in a direct accretion state mapping, then the radio/X-ray correlations observed in the so-called "hard state" of BHBs would not be expected to apply to Seyferts, which are in a radio-quiet state more similar to the "soft state" of BHBs. It is thus rather strange that Seyferts land so close to the correlation at all. When they are excluded, the scatter is significantly reduced (Körding et al. 2006; Gültekin et al. 2009), while samples of BL Lacs adhere to the correlation very well (see Plotkin et al., this volume). Newer work including lower luminosity Seyferts (King, Miller et al., in prep.) may indicate a separate correlation for Seyferts corresponding to their expected higher accretion efficiency, but this is clearly a point that still needs to be resolved.

The FP is generally understood to be driven by ratios of radiative efficiencies (see, e.g., Markoff et al. 2003; Heinz \& Sunyaev 2003), but there is still significant degeneracy in understanding the exact physical drivers. For instance, different X-ray producing mechanisms ranging from direct synchrotron, synchrotron self-Compton and inverse Compton from radiatively inefficient flows can all account reasonably for the correlations observed. Thus to move beyond simple scalings, it is desirable to test physical models of the broadband spectra that can scale with mass.

\section{Results}

We first tried this test a few years ago for the nearby low-luminosity AGN M81, using an outflow dominated model originally fit to many different observations of hard state BHBs (Markoff et al. 2005; Gallo et al. 2007; Migliari et al. 2007; Maitra et al. 2009). In the BHB fits, the main free parameters were the input power $N_{j}$ in $L_{\text {Edd }}$ units, the size of the jet base/corona $R_{0}$ (in mass-scaling units of $r_{g}$ ), the temperature of the assumed thermal distribution of electrons injected at the base, $T_{e}$, the location of the start of particle acceleration in the jets $z_{\text {acc }}$, the power law index of the accelerated particle tail $p$ and the ratio of magnetic to internal energy density $k$. Another parameter included in the model is the inner edge of the accretion disk $R_{\text {in }}$. The accretion disk cannot often be directly constrained by the fits, and contrary to what occurs with thermal Comptonization models, it is not the only contributor of the upscattered photon field. However it does play a role, and the inner radius can be somewhat constrained by the X-ray fit, though it is not part of the core model. While $N_{j}$ varied appropriate to the luminosity of the observations, most parameters were found to fall in rather small ranges for a variety of sources.

When we applied the model used for the BHBs to our simultaneous radio through X-ray data from a coordinated campaign on M81 (Markoff et al. 2008), we found that the best fit yielded parameter values in exactly the same range as that found for the stellar-mass sources. The only exception was the jet base/corona temperature $T_{e}$, which was $2-5$ times hotter in M81. We are currently investigating the reality of assuming a thermal distribution in the first place (Pe'er, Markoff et al., in prep.) but one explanation is that the (in our case, non-bound) plasma cools much faster in the denser environment near a stellar-mass black hole, with the same relative accretion rate $\dot{m}$ fed into the same scaled size in $r_{g}$ units.

In this article we present our newest attempt to test the underlying physics of the FP, by performing the homogeneous modeling of two sources separated by over six orders of magnitude in mass. If the FP is truly driven by shared physics as a function of $\dot{m}$, then 


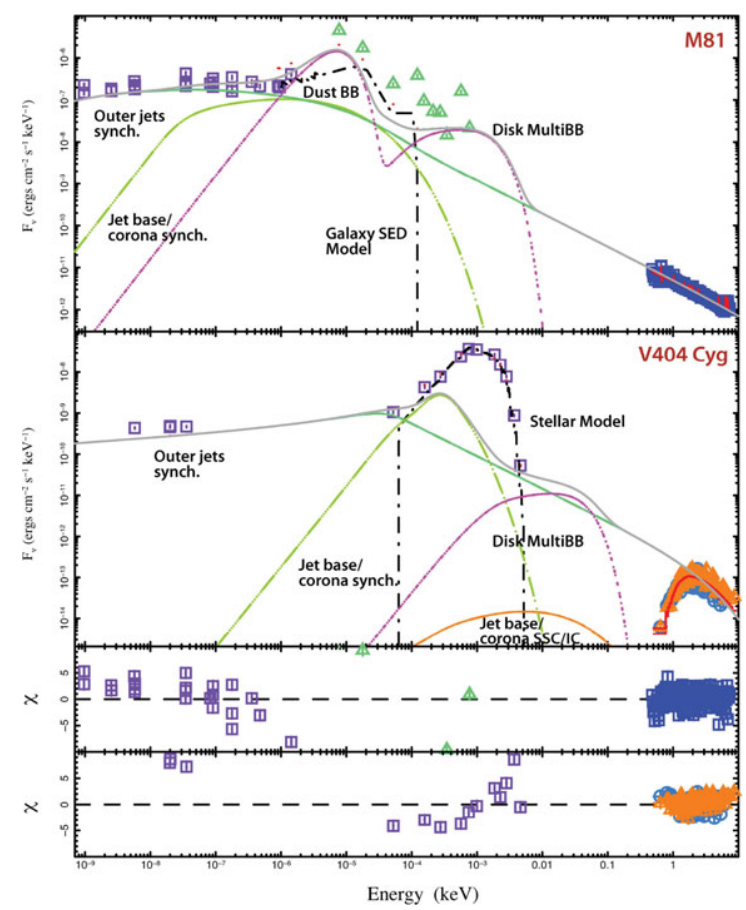

Figure 1. First attempted joint modeling of an AGN and BHB, in this case M81 and V404 Cyg (see Markoff et al. 2008; Hynes et al. 2009 for details about the data sets). The same physical model, based on Markoff et al. (2005), was applied to both sources, with individual physical parameters such as mass, distance and inclination fixed at values determined from observations. The free parameters $R_{0}, p, z_{\text {acc }}, k$ and $R_{\text {in }}$ (see text for explanation) were fixed to vary together for the two sources. Additional components for star/stars, and dust components dominate the opt/IR range. This new approach, although preliminary, already results in promising fits to both data sets.

two accreting BHs at roughly the same scaled power should be well explained by the same physical model, if all size and power scales are expressed in mass-scaling units $\left(r_{g}\right.$, and $\dot{m}$ or $\left.L_{\mathrm{Edd}}\right)$.

The difficulty is in identifying two sources with the same $\dot{m}$, since part of the degeneracy in the models involves uncertainty about the relationship between $L_{\mathrm{bol}}$ or $L_{\mathrm{X}}$ and $\dot{M}$, as well as uncertainties in the actual BH mass. M81 is a good source to start with on the supermassive side, because its mass and distance are very well constrained, as well as the availability of a high-quality simultaneous broadband spectrum (Markoff et al. 2008). In our campaign we found M81 to be in a relatively low state compared to its prior average, at $L_{\mathrm{X}} \sim 2 \times 10^{-6}$, about an order of magnitude lower than some earlier epochs. For the BHB we chose V404 Cyg, specifically a simultaneous multiwavelength data set during quiescence presented in Hynes et al. (2009). The source was about 10 times fainter in the X-rays, in Eddington units, compared to the observation of M81. On the other hand, the FP correlation slope tells us that $L_{\mathrm{X}} \propto \dot{M}^{2-2.4}$ (Markoff et al. 2003; Heinz \& Sunyaev 2003), so the difference in $\dot{m}$ is only a factor of a few. We felt that this was close enough to assess whether the same mass-scaled model could apply to two sources at vastly different physical scales.

In this proceedings we present a very preliminary attempt, with all the caveats that entails because we have not yet had time to explore parameter space, but it is already 
quite interesting. The core model used is the outflow dominated model developed in Markoff et al. (2005) and Maitra et al. (2009), supplemented for M81 with a plasma model for the X-ray line emission as described in Young et al. (2007), and with a stellar model for V404 Cyg presented in Hynes et al. (2009). Further, the IR/optical data for M81 are non-simultaneous and include some contamination from the galaxy stellar population and dust, so we are exploring models which combine nuclear emission with additional galactic components in this region. For the key parts of the spectrum as far as the FP is concerned, the radio and the X-ray bands, clearly a reasonable solution has been found that can explain both the SEDs as well as the broadband correlations. In the model presented here, both sources are dominated by synchrotron emission in the X-ray band, which is compatible with recent results by Russell et al. (2010), but other solutions may yet be found.

\section{Discussion}

We are currently exploring the parameter space for this new class of joint models, to see which combinations of tied parameters result in the most sensible fits across the mass scale (Markoff, Nowak et al., in prep.). This approach offers interesting, new possibilities for exploring the interplay of accretion physics and the environment. For instance, one could use joint models with appropriate microquasars to study the effect of the environment in the FRI/FRII differentiation. The technique could also be exploited to help "fill" the gaps in multiwavelength campaigns where coverage was limited, to help make predictions for further observations, or to constrain models. At the very lowest accretion rates, where weak luminosities and small count rates lead to poor statistics, a combined fit with a closer and thus brighter source of comparable accretion rate could be an invaluable new strategy.

\section{References}

Corbel, S., Nowak, M., Fender, R. P., Tzioumis, A. K., \& Markoff, S. 2003, A\& A, 400, 1007

Falcke, H., Körding, E., \& Markoff, S. 2004, A\&SA, 414, 895

Gallo, E., Migliari, S., Markoff, S., Tomsick, J. A., Bailyn, C. D., Berta, S., Fender, R., \& Miller-Jones, J. C. A. 2007, ApJ, 670, 600

Gültekin, K., Cackett, E. M., Miller, J. M., Di Matteo, T., Markoff, S., \& Richstone, D. O. 2009, ApJ, 706, 404

Heinz, S. \& Sunyaev, R. A. 2003, MNRAS, 343, L59

Hynes, R. I., Bradley, C. K., Rupen, M., Gallo, E., Fender, R. P., Casares, J., \& Zurita, C. 2009, MNRAS, 399, 2239

Körding, E., Falcke, H., \& Corbel, S. 2006, A\&A, 456, 439

Maitra, D., Markoff, S., Brocksopp, C., Noble, M., Nowak, M., \& Wilms, J. 2009, MNRAS, 398, 1638

Markoff, S., Nowak, M., Corbel, S., Fender, R., \& Falcke, H. 2003, A\&SA, 397, 645

Markoff, S., Nowak, M., Young, A., Marshall, H. L., Canizares, C. R., Peck, A., Krips, M., Petitpas, G., Schödel, R., Bower, G. C., Chandra, P., Ray, A., Muno, M., Gallagher, S., Hornstein, S., \& Cheung, C. C. 2008, ApJ, 681, 905

Markoff, S., Nowak, M. A., \& Wilms, J. 2005, ApJ, 635, 1203

Merloni, A., Heinz, S., \& di Matteo, T. 2003, MNRAS, 345, 1057

Migliari, S., Tomsick, J. A., Markoff, S., Kalemci, E., Bailyn, C. D., Buxton, M., Corbel, S., Fender, R. P., \& Kaaret, P. 2007, ApJ, 670, 610

Russell, D. M., Maitra, D., Dunn, R. J. H., \& Markoff, S. 2010, MNRAS, 405, 1759

Young, A. J., Nowak, M. A., Markoff, S., Marshall, H. L., \& Canizares, C. R. 2007, ApJ, 669, 830 


\section{Discussion}

Proga: What is the value of $T_{e} / T_{i}$ in your spectrum fitting method?

MARKOFF: At the moment it's set $T_{e}=T_{i}$, but in the future simulations we can play with that parameter.

ProgA: Can you determine this from the spectral fits from your simulations?

MARKOFF: We just started doing these, so it's something we will look into in the near future.

Bosch-RAmon: You may include thermal free-free emission in your models to get a better estimate of the proton temperature ratio.

MARKOFF: For Sgr $\mathrm{A}^{*}$, free-free emission from the inner part we simulate is negligible, but for the scaling models applied to XRB/AGN, we plan to include protons and hadronic interactions, but it is not yet in the model. 